\title{
Risk of subsequent stroke, with or without extracranial-intracranial bypass surgery: a nationwide, retrospective, population-based study
}

\author{
XianXiu Chen, MPH, PhD, ${ }^{1,2}$ Cheng-Li Lin, MSc, ${ }^{3,4}$ Yuan-Chih Su, MSc, ${ }^{3,4}$ Kuan-Fei Chen, MD, ${ }^{5}$ \\ Shih-Wei Lai, MD, ${ }^{3,6}$ Sung-Tai Wei, MD, ${ }^{7}$ Ching-Tien Peng, MD, ${ }^{8,9}$ Cheng-Di Chiu, MD, PhD, 2,7,10 \\ Shwn-Huey Shieh, PhD, ${ }^{11}$ and Chun-Chung Chen, MD2,3,7

\begin{abstract}
${ }^{1}$ Department of Public Health, China Medical University; ${ }^{2}$ Stroke Center, China Medical University Hospital; ${ }^{3}$ College of Medicine, China Medical University; ${ }^{4}$ Management Office for Health Data, China Medical University Hospital; ${ }^{5}$ Department of of Neurosurgery, China Medical University Hospital; ${ }^{8}$ Department of Hemato-oncology, Children's Hospital, China Medical University; ' Department of Biotechnology, Asia University; ${ }^{10} \mathrm{Graduate}$ Institute of Basic Medical Science, China Medical University; and ${ }^{11}$ Department of Health Services Administration, China Medical University, Taichung, Taiwan
\end{abstract} \\ Neurology, China Medical University Hospital; ${ }^{6}$ Department of Family Medicine, China Medical University Hospital; ${ }^{7}$ Department
}

\begin{abstract}
OBJECTIVE Although no benefits of extracranial-intracranial (EC-IC) bypass surgery in preventing secondary stroke have been identified previously, the outcomes of initial symptomatic ischemic stroke and stenosis and/or occlusion among the Asian population in patients with or without bypass intervention have yet to be discussed. The authors aimed to evaluate the subsequent risk of secondary vascular disease and cardiac events in patients with and without a history of this intervention.
\end{abstract}

METHODS This retrospective nationwide population-based Taiwanese registry study included 205,991 patients with initial symptomatic ischemic stroke and stenosis and/or occlusion, with imaging data obtained between 2001 and 2010. Patients who underwent EC-IC bypass (bypass group) were compared with those who had not undergone EC-IC bypass, carotid artery stenting, or carotid artery endarterectomy (nonbypass group). Patients with any previous diagnosis of ischemic or hemorrhagic stroke, moyamoya disease, cancer, or trauma were all excluded.

RESULTS The risk of subsequent ischemic stroke events decreased by $41 \%$ in the bypass group (adjusted hazard ratio $[\mathrm{HR}] 0.59,95 \% \mathrm{Cl} 0.46-0.76, \mathrm{p}<0.001$ ) compared with the nonbypass group. The risk of subsequent hemorrhagic stroke events increased in the bypass group (adjusted HR 2.47, 95\% Cl 1.67-3.64, $p<0.001$ ) compared with the nonbypass group.

CONCLUSIONS Bypass surgery does play an important role in revascularization of the ischemic brain, while also increasing the risk of hemorrhage in the early postoperative period. This study highlights the fact that the high risk of bypass surgery obscures the true benefit of revascularization of the ischemic brain and also emphasizes the importance of developing improved surgical technique to treat these high-risk patients.

https://thejns.org/doi/abs/10.3171/2017.12.JNS172178

KEYWORDS secondary prevention; revascularization; cerebrovascular procedures; ischemic stroke; EC-IC bypass; extracranial-intracranial bypass; vascular disorders

$\mathrm{S}$ OME ischemic strokes are hemodynamic in nature (rather than thromboembolic), with progressively diminishing cerebral blood flow in the absence of compensatory collateral circulation. ${ }^{13}$ The annual rate of hemodynamic ischemic stroke is $25 \%$, which is estimated to increase by $2 \%$ annually, and patients with this stroke typically fail to respond to medical treatment. ${ }^{2}$ Complete occlusion of the internal carotid artery (ICA) occurs in $5 \%-15 \%$ of patients with cerebral infarctions due to transient ischemia in the carotid territory. Rates of the 2-year risk of recurrent stroke following medical therapy are 5\%$9 \%$ per year for all types of stroke and $2 \%-8 \%$ per year for

ABBREVIATIONS AMI = acute myocardial infarction; $\mathrm{CHF}=$ congestive heart failure; EC-IC = extracranial-intracranial; HR = hazard ratio; ICA = internal carotid artery; ICD-9-CM = International Classification of Diseases, Ninth Revision, Clinical Modification; MCA = middle cerebral artery; NHIRD = National Health Insurance Research Database; STA = superficial temporal artery.

SUBMITTED August 31, 2017. ACCEPTED December 4, 2017.

INCLUDE WHEN CITING Published online June 1, 2018; DOI: 10.3171/2017.12.JNS172178. 
ipsilateral ischemic stroke. ${ }^{8,10,14,15}$ Patients with evidence of hemodynamic compromise on functional imaging are at an even higher risk. The rate of recurrent stroke in patients with occlusion of the ICA is $4.8 \%$ at 1 year, $12.2 \%$ at 3 years, and $17.1 \%$ at 5 years after the index event. ${ }^{18}$

Cerebral bypass surgery is an option for carefully selected patients and involves revascularization of the cerebral arteries through extracranial grafts, improving the hemodynamic status of the circulation distal to the occluded vessel. ${ }^{3,21,22}$ Cerebral artery bypass reroutes blood flow to improve or to restore blood flow around a blocked or damaged artery to an ischemic area of the brain. Depending on the site of the blockage, the underlying condition being treated, and the size of the brain area, cerebral bypass can be performed in many ways for revascularization. ${ }^{24}$ Superficial temporal artery-middle cerebral artery (STA-MCA) bypass is the most commonly performed type of bypass. Blood flow through the MCA is often reduced with ICA narrowing. In STA-MCA bypass, the STA is rerouted from the scalp through a hole in the skull and connected to the MCA to restore the blood flow to the brain.,24 Thus, this surgery is also called extracranial-intracranial (EC-IC) bypass. ${ }^{2}$ This technique has been extensively developed since the first EC-IC arterial anastomosis was performed in $1967,6,7$ but its use remains controversial., $9,12,16,19,23$

Identifying the patients who can benefit from EC-IC bypass surgery has proved to be daunting. ${ }^{5} \mathrm{~A}$ recently published randomized trial comparing the procedure to medical therapy found that the surgery was associated with a lower rate of stroke from the end of the 30-day perioperative period to 2 years postrandomization (6\% and $18 \%$, respectively). Yet, the surgery did not reduce the overall stroke rate from randomization to 2 years later because of a higher stroke rate during the perioperative period $(14 \%$ in the 30 days immediately postrandomization vs $2 \%$ for medical therapy). ${ }^{17}$ An earlier investigation (the Carotid Occlusion Surgery Study) also failed to show any benefit of EC-IC surgery, thus negatively influencing the opinion about surgical treatment for ischemic cerebrovascular disease. ${ }^{17}$ However, that study has been widely criticized, predominantly because of patient selection bias. ${ }^{7,20}$

Although previous reports have not identified a benefit of this procedure, the outcomes of initial symptomatic stenosis and/or occlusion in patients with ischemic stroke with or without bypass intervention in an Asian population have yet to be discussed. Herein, we evaluated the subsequent risk of secondary vascular disease and cardiac events in inpatients with ischemic stroke with and without EC-IC intervention, using data from the National Health Insurance Research Database (NHIRD; https://nhird.nhri. org.tw/en/) of Taiwan. The NHIRD has covered more than $99 \%$ of the entire Taiwanese population over the last 2 decades, with patient-level medical records systematically compiled and stored.

\section{Methods}

\section{Study Design}

This retrospective, nationwide, population-based, Taiwanese registry cohort study used data from the years 2000-2013 (study period) from the NHIRD of Taiwan.
Clinical diagnoses were coded using the International Classification of Diseases, Ninth Revision, Clinical Modification (ICD-9-CM).

The study population consisted of all inpatients hospitalized between January 1, 2001, and December 31, 2010 (enrollment period; the length of time between the study period and the enrollment period was left out for purposes of medical history and follow-up investigation), 1) with a diagnosis of initial ischemic stroke, with stenosis and/or occlusion (ICD-9-CM 433-434), which was defined as the index event; and 2) who underwent CT or MRI examination within 2 days before or after the hospitalization date (Fig. 1A). Patients who met these inclusion criteria and underwent EC-IC bypass after the index event were categorized as the bypass group, and those who met the criteria but had never undergone EC-IC bypass, carotid artery stenting, or carotid artery endarterectomy after the index event were defined as the nonbypass group. The date of initial ischemic stroke was defined as the index date for the nonbypass group. In contrast, to exclude immortal time bias, the date of EC-IC bypass was defined as the index date for the bypass group. Patients with any diagnosis of ischemic stroke (ICD-9-CM 433-435), hemorrhagic stroke (ICD-9-CM 430-432), or moyamoya disease (ICD-9-CM 437.5) before the index event were excluded. We also excluded those with any record of cancer (ICD-9-CM 140-208) or trauma (ICD-9-CM 36565, 3662x, 37647, 37652, 38051, 3811, 5185, 5981, 6648x, 6649x, 665, 6658x, 6659x, 7161, 7217, 72812, 860x, 878x, 885x, 886x, 887x, 895x, 896x, 897x, 958, 9584, $9585,9587,9588$ ) before and after the index event.

The outcome factors included symptomatic ischemic stroke with stenosis and/or occlusion, hemorrhagic stroke, and mortality after the index date. Cardiac events, which included congestive heart failure (CHF; ICD-9-CM 428) and acute myocardial infarction (AMI; ICD-9-CM 410) that occurred during the study period, were also evaluated (Fig. 1B).

\section{Data Source}

The NHIRD is population-based and derived from the claims data of the National Health Insurance program, which is a mandatory-enrollment, single-payer system created in 1995 that now covers more than $99 \%$ of Taiwan's population (https://nhird.nhri.org.tw/en/). ${ }^{11}$ The NHIRD has been used widely by researchers in Taiwan; the data have been found to be highly accurate in the recording of an ischemic stroke diagnosis, and the database has acceptable validity for epidemiological research on ischemic stroke. ${ }^{4}$ To protect personal information, patient data were de-identified before the inpatient database was released for research purposes. Thus, the requirement for informed patient consent was waived for the present study. This study was approved by the institutional review board of the China Medical University and Hospital in Taiwan.

\section{Statistical Methods}

The incidence rate of each outcome was calculated in terms of person-days. The hazard ratio (HR) and 95\% CI for the risk of each outcome were estimated in univariable and multivariable Cox proportional hazards regression 

A Inclusion Criteria:
Inpatients with first claims of ischemic stroke and stenosis-occlusion, with CT or MR data available (index event).
( $\mathrm{n}=205991$ )
Study Period: 2000-2013; Enrolled Period: 2001-2010

\section{凹}

\section{Study population:}
1. Case Cohort: Meets the inclusion criteria, and EC-IC bypass performed after the index event ( $n=2156$ ) (index date: bypass performed date).
2. Matched Cohort: Meets the inclusion criteria, and did not undergo ECIC bypass, carotid artery stenting, or carotid artery endarterectomy after the index event $(n=200394)$ (index date: index event date).

FIG. 1. Schema and flow chart of the study. A: Flow chart of the study. Interference of the measured confounding factors was avoided by performing a 1:4 casecontrol match using a propensity score. B: Schema of the study. The index date was defined as the date of surgery for patients who underwent surgical intervention. For the control group, the index date was defined as the date of hospitalization after stroke onset. This was done to eliminate the immortal time bias. AF = atrial fibrillation; CKD = chronic kidney disease; COPD = chronic obstructive pulmonary disease; $\mathrm{DM}=$ diabetes mellitus.

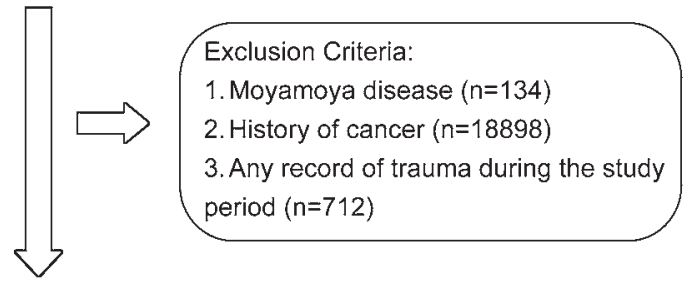

Final valid subject:

1. Case Cohort: Inpatients with first claim of ischemic stroke and stenosis-occlusion, with CT or MR data available, and EC-IC bypass performed after the hospitalization $(n=1440)$. 2. Matched Cohort: Inpatients with first claim of ischemic stroke and stenosis-occlusion, with CT or MR data available, and who did not undergo ECIC bypass, carotid artery stenting, or carotid artery endarterectomy after the hospitalization $(n=104179)$.

\section{乙}

Matching: 1:4 propensity score match by sex, age, the year of index event and index

date, and comorbidities

1. Case Cohort: bypass group ( $n=1440)$

2. Matched Cohort: no bypass group $(n=5760)$

B Exclusion Criteria:

moyamoya and all cancers before index date. And trauma within the study period.

Matching medical history:

congestive heart failure, hypertension, AF, hyperlipidemia, DM, COPD, CKD

Event:

1. Secondary stroke occurred within 1 month after index event

2. Death within 1 month after index event.

3. Cardiac event during the study period.

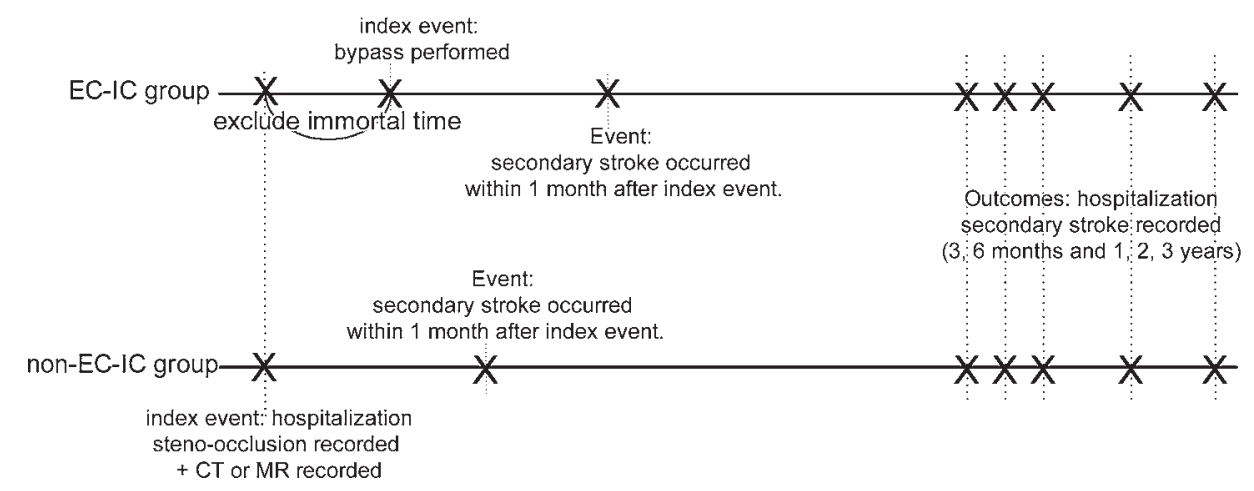


TABLE 1. Demographic data in the study population (patients with ischemic stroke)

\begin{tabular}{|c|c|c|c|c|c|}
\hline \multirow[b]{3}{*}{ Characteristic } & \multicolumn{4}{|c|}{ Bypass } & \multirow{3}{*}{$\begin{array}{c}p \\
\text { Value }\end{array}$} \\
\hline & \multicolumn{2}{|c|}{ No, $n=5760$} & \multicolumn{2}{|c|}{ Yes, $n=1440$} & \\
\hline & No. & $\%$ & No. & $\%$ & \\
\hline Sex & & & & & 0.95 \\
\hline Female & 2029 & 35.2 & 506 & 35.1 & \\
\hline Male & 3731 & 64.8 & 934 & 64.9 & \\
\hline Age, yrs & & & & & 0.19 \\
\hline$<35$ & 125 & 2.17 & 26 & 1.81 & \\
\hline $35-64$ & 2444 & 42.4 & 647 & 44.9 & \\
\hline$\geq 65$ & 3191 & 55.4 & 767 & 53.3 & \\
\hline Mean $(\mathrm{SD})^{*}$ & \multicolumn{2}{|c|}{$66.8(14.5)$} & \multicolumn{2}{|c|}{$64.4(12.8)$} & \\
\hline \multicolumn{6}{|l|}{ Baseline comorbidity } \\
\hline CAD & 1042 & 18.1 & 263 & 18.3 & 0.88 \\
\hline $\mathrm{CHF}$ & 857 & 14.9 & 245 & 17.0 & 0.04 \\
\hline Hypertension & 3303 & 57.3 & 840 & 58.3 & 0.50 \\
\hline Atrial fibrillation & 508 & 8.82 & 142 & 9.86 & 0.22 \\
\hline Hyperlipidemia & 1102 & 19.1 & 290 & 20.1 & 0.39 \\
\hline Diabetes mellitus & 1728 & 30 & 444 & 30.8 & 0.54 \\
\hline COPD & 420 & 7.29 & 107 & 7.43 & 0.86 \\
\hline CKD & 159 & 2.76 & 51 & 3.54 & 0.12 \\
\hline Stroke w/in 1 mo of index date & & & & & $<0.001$ \\
\hline Ischemic stroke (ICD-9-CM 433 \& 434) & 42 & 0.73 & 4 & 0.28 & \\
\hline Hemorrhagic stroke (ICD-9-CM 430-432) & 7 & 0.12 & 10 & 0.69 & \\
\hline Mortality w/in 1 mo of index date & 373 & 6.48 & 147 & 10.2 & $<0.001$ \\
\hline CHF event w/in study period & 299 & 5.19 & 114 & 7.92 & $<0.001$ \\
\hline AMI event w/in study period & 160 & 2.78 & 19 & 1.32 & $<0.001$ \\
\hline Mean follow-up period in yrs (median) & \multicolumn{2}{|c|}{$3.34(2.45)$} & \multicolumn{2}{|c|}{$3.34(2.09)$} & 0.002 \\
\hline Duration of stroke to bypass in days $(95 \% \mathrm{Cl})$ & & & \multicolumn{2}{|c|}{$690.1(641.4-738.7)$} & \\
\hline
\end{tabular}

models. To avoid the interference of measured confounding factors, we performed a 1:4 case-control match with propensity score matching that was obtained from logistic regression analysis of the bypass intervention on sex, age, year of index event and index date, and comorbidities. The comorbidities included coronary artery disease (ICD9-CM 411-414), congestive heart failure (CHF; ICD9-CM 428), hypertension (ICD-9-CM 401-405), atrial fibrillation (ICD-9-CM 427.31), hyperlipidemia (ICD-9CM 272.0-272.4), diabetes mellitus (ICD-9-CM 250), chronic obstructive pulmonary disease (ICD-9-CM 491, 492, 496), and chronic kidney disease (ICD-9-CM 250.4, 274.1, 283.11, 403, 404, 440.1, 442.1, 447.3, 572.4, 580$588,642.1,646.2)$. The nearest-neighbor matching method was used in the propensity model. Statistical analyses were performed using SAS statistical software (version 9.4 for Windows; SAS Institute, Inc.). The significance level was set at 0.05 , and all tests were 2 -tailed.

\section{Results}

A total of 205,991 first claims for ischemic stroke and stenosis and/or occlusion in patients with available CT or
MR data were identified during the enrollment period. Of these, 2156 patients underwent EC-IC bypass after the index event; 200,394 patients did not undergo EC-IC bypass, and 3441 who ever underwent either carotid artery stenting, carotid artery endarterectomy, or more than 2 interventions after the index event were excluded. After the exclusion of those who did not meet the inclusion criteria, a total of 105,619 patients were analyzed (Fig. 1A). Table 1 presents the baseline characteristics of the study groups, baseline comorbidities, time duration from stroke to bypass, information regarding recurrent stroke events and mortality within 1 month after the index date, and the cardiac events during the study period. There were statistically significant group differences in medical history of CHF and cardiac events during the study period, recurrent stroke events, and mortality within 1 month after the index date. Table 2 presents the crude and adjusted HRs and 95\% CIs for the conditional logistic regression analyses.

The risk of subsequent strokes of all types (ischemic and hemorrhagic; crude HR 0.8, 95\% CI 0.65-0.99) was significantly lower in the bypass group compared with that in the nonbypass group $(\mathrm{p}<0.05)$. After adjustment for 
TABLE 2. Incidence and risk of recurrent stroke, stratified by follow-up period

\begin{tabular}{|c|c|c|c|c|c|c|c|c|}
\hline \multirow[b]{3}{*}{ Follow-Up Period } & \multicolumn{6}{|c|}{ Ischemic Stroke } & \multicolumn{2}{|c|}{ Bypass vs Nonbypass } \\
\hline & \multicolumn{3}{|c|}{ Nonbypass } & \multicolumn{3}{|c|}{ Bypass } & \multirow{2}{*}{$\begin{array}{l}\text { Crude HR } \\
(95 \% \mathrm{Cl})\end{array}$} & \multirow{2}{*}{$\begin{array}{c}\text { Adjusted HR } \\
(95 \% \mathrm{Cl})\end{array}$} \\
\hline & Event No. & PY & Rate & Event No. & PY & Rate & & \\
\hline \multicolumn{9}{|l|}{ Overall, all stroke } \\
\hline Overall & 555 & 19,228 & 2.89 & 109 & 4814 & 2.26 & $0.8(0.65-0.99)^{*}$ & $0.84(0.68-1.03)$ \\
\hline$\leq 3 \mathrm{mos}$ & 49 & 750 & 6.53 & 13 & 176 & 7.38 & $1.13(0.61-2.08)$ & $1.12(0.61-2.07)$ \\
\hline$>3-\leq 6 \mathrm{mos}$ & 41 & 1039 & 3.94 & 15 & 242 & 6.21 & $1.57(0.87-2.84)$ & $1.66(0.91-3.02)$ \\
\hline$>6-\leq 12 \mathrm{mos}$ & 76 & 1892 & 4.02 & 12 & 437 & 2.75 & $0.68(0.37-1.26)$ & $0.76(0.41-1.41)$ \\
\hline$>1-\leq 2$ yrs & 108 & 3305 & 3.27 & 7 & 777 & 0.9 & $0.28(0.13-0.59)^{\star \star *}$ & $0.29(0.13-0.62)^{\star *}$ \\
\hline$>2-\leq 3$ yrs & 66 & 2861 & 2.31 & 15 & 681 & 2.2 & $0.95(0.55-1.67)$ & $0.96(0.55-1.7)$ \\
\hline$>3 \mathrm{yrs}$ & 166 & 8961 & 1.85 & 33 & 2399 & 1.38 & $0.75(0.52-1.09)$ & $0.78(0.54-1.14)$ \\
\hline \multicolumn{9}{|l|}{ Ischemic stroke } \\
\hline Overall & 488 & 19,228 & 2.54 & 68 & 4814 & 1.41 & $0.57(0.44-0.73)^{\star * *}$ & $0.59(0.46-0.76)^{* * *}$ \\
\hline$\leq 3 \mathrm{mos}$ & 46 & 750 & 6.13 & 7 & 176 & 3.97 & $0.65(0.29-1.44)$ & $0.64(0.29-1.43)$ \\
\hline$>3-\leq 6 \mathrm{mos}$ & 32 & 1039 & 3.08 & 7 & 242 & 2.9 & $0.94(0.41-2.13)$ & $0.94(0.41-2.15)$ \\
\hline$>6-\leq 12 \mathrm{mos}$ & 64 & 1892 & 3.38 & 4 & 437 & 0.92 & $0.27(0.1-0.74)^{*}$ & $0.31(0.11-0.84)^{*}$ \\
\hline$>1-\leq 2$ yrs & 97 & 3305 & 2.93 & 6 & 777 & 0.77 & $0.26(0.12-0.6)^{* *}$ & $0.27(0.12-0.63)^{* *}$ \\
\hline$>2-\leq 3$ yrs & 55 & 2861 & 1.92 & 14 & 681 & 2.05 & $1.07(0.59-1.92)$ & $1.09(0.6-1.98)$ \\
\hline$>3 \mathrm{yrs}$ & 148 & 8961 & 1.65 & 26 & 2399 & 1.08 & $0.66(0.44-1.01)$ & $0.69(0.45-1.05)$ \\
\hline \multicolumn{9}{|l|}{ Hemorrhagic stroke } \\
\hline Overall & 71 & 19,228 & 0.37 & 41 & 4814 & 0.85 & $2.39(1.63-3.51)^{\star * *}$ & $2.47(1.67-3.64)^{\star * *}$ \\
\hline$\leq 3 \mathrm{mos}$ & 3 & 750 & 0.4 & 6 & 176 & 3.4 & $8.52(2.13-34.06)^{\star *}$ & $8.66(2.12-35.37)^{\star *}$ \\
\hline$>3-\leq 6 \mathrm{mos}$ & 9 & 1039 & 0.87 & 8 & 242 & 3.31 & $3.82(1.48-9.91)^{\star *}$ & $4.84(1.81-12.9)^{* *}$ \\
\hline$>6-\leq 12 \mathrm{mos}$ & 12 & 1892 & 0.63 & 8 & 437 & 1.83 & $2.89(1.18-7.06)^{*}$ & $3.13(1.26-7.81)^{*}$ \\
\hline$>1-\leq 2 \mathrm{yrs}$ & 11 & 3305 & 0.33 & 1 & 777 & 0.13 & $0.39(0.05-3)$ & $0.41(0.05-3.21)$ \\
\hline$>2-\leq 3 \mathrm{yrs}$ & 11 & 2861 & 0.38 & 1 & 681 & 0.15 & $0.38(0.05-2.96)$ & $0.38(0.05-3)$ \\
\hline$>3 \mathrm{yrs}$ & 18 & 8961 & 0.2 & 7 & 2399 & 0.29 & $1.48(0.62-3.55)$ & $1.53(0.63-3.71)$ \\
\hline
\end{tabular}

$\mathrm{PY}=$ person-year; rate $=$ recurrence rate (per 100 person-years).

Adjusted HR denotes adjusted for sex, age, and comorbidities in Cox proportional hazards regression.

${ }^{*} p<0.05 ;{ }^{* *} p<0.01 ;{ }^{* * *} p<0.001$.

possible confounders (age, sex, follow-up period, and comorbidities), the risk of recurrent strokes of all types (adjusted HR 0.84, 95\% CI 0.68-1.03) slightly increased from that in the nonbypass group, but without significant difference. In general, the risk of recurrent strokes of all types was higher until 6 months postoperatively in the bypass group than that in the nonbypass group (without statistical significance), whereas the risk of recurrent strokes of all types showed a decreasing trend 6 months after the index date in the bypass group compared with the nonbypass group. The significant differences were seen after $>1$ to $\leq$ 2 years (crude HR $0.28,95 \%$ CI $0.13-0.59, \mathrm{p}<0.001$ and adjusted HR 0.29, 95\% CI 0.13-0.62, p < 0.01).

Subsequent ischemic stroke occurred in $488 / 5760 \mathrm{pa}-$ tients (incidence rate 2.54) in the nonbypass group and in $68 / 1440$ patients (incidence rate 1.41) in the bypass group (crude HR 0.57, 95\% CI 0.44-0.73, p < 0.001). After adjustment for possible confounders, the risk of subsequent ischemic stroke events was lowered by $41 \%$ in the bypass group (adjusted HR 0.59, 95\% CI 0.46-0.76, p < 0.001) relative to that in the nonbypass group. In general, the risk of subsequent ischemic stroke was lower in the bypass group than in the nonbypass group, with a significant dif- ference between 6 months and 2 years after the index date ( $>6$ to $\leq 12$ months: adjusted HR $0.31,95 \%$ CI $0.11-0.84$, $\mathrm{p}<0.05 ;>1$ to $\leq 2$ years: adjusted HR $0.27,95 \%$ CI 0.12 $0.63, \mathrm{p}<0.01)$.

Subsequent hemorrhagic stroke occurred in $71 / 5760 \mathrm{pa}-$ tients (incidence rate 0.37 ) in the nonbypass group and in $41 / 1440$ patients (incidence rate 0.85 ) in the bypass group (crude HR 2.39, 95\% CI 1.63-3.51, p < 0.001). After adjustment for possible confounders, the risk of subsequent hemorrhagic stroke events increased in the bypass group (adjusted HR 2.47, 95\% CI 1.67-3.64, p < 0.001) relative to that in the nonbypass group. In general, the risk of subsequent hemorrhagic stroke was higher in the bypass group than in the nonbypass group, with significant difference before 1 year postsurgery or stroke ( $\leq 3$ months: adjusted HR 8.66, 95\% CI 2.12-35.37, $\mathrm{p}<0.01 ;>3$ to $\leq 6$ months: adjusted HR 4.84, 95\% CI 1.81-12.9, p < 0.01; and $>6$ to $\leq$ 12 months: adjusted HR 3.13, 95\% CI 1.26-7.81, p < 0.05).

Table 3 presents the mortality data postsurgery or stroke. Relative to that in the nonbypass group, the risk of death was slightly higher in the bypass group, but without significant difference. In general, the risk of subsequent mortality was higher in the bypass group than in the non- 
TABLE 3. Mortality, stratified by follow-up period

\begin{tabular}{|c|c|c|c|c|c|c|c|c|}
\hline \multirow{3}{*}{$\begin{array}{c}\text { Follow-Up } \\
\text { Period }\end{array}$} & \multicolumn{6}{|c|}{ Ischemic Stroke } & \multicolumn{2}{|c|}{ Bypass vs Nonbypass } \\
\hline & \multicolumn{3}{|c|}{ Nonbypass } & \multicolumn{3}{|c|}{ Bypass } & \multirow{2}{*}{$\begin{array}{c}\text { Crude HR } \\
(95 \% \mathrm{Cl})\end{array}$} & \multirow{2}{*}{$\begin{array}{c}\text { Adjusted HR } \\
(95 \% \mathrm{Cl})\end{array}$} \\
\hline & Event No. & PY & Rate & Event No. & PY & Rate & & \\
\hline \multicolumn{9}{|l|}{ Mortality } \\
\hline Overall & 1587 & 20,622 & 7.7 & 511 & 5173 & 9.88 & $1.31(1.18-1.44)^{* * *}$ & $1.35(1.22-1.49)^{* * *}$ \\
\hline$\leq 3 \mathrm{mos}$ & 169 & 757 & 22.32 & 40 & 180 & 22.25 & $1(0.71-1.41)$ & $1.01(0.72-1.43)$ \\
\hline$>3-\leq 6 \mathrm{mos}$ & 113 & 1055 & 10.71 & 41 & 249 & 16.44 & $1.53(1.07-2.19)^{*}$ & $1.83(1.27-2.63)^{* *}$ \\
\hline$>6-\leq 12 \mathrm{mos}$ & 144 & 1942 & 7.41 & 48 & 454 & 10.57 & $1.43(1.03-1.98)^{*}$ & $1.62(1.16-2.25)^{* *}$ \\
\hline$>1-\leq 2 \mathrm{yrs}$ & 196 & 3455 & 5.67 & 64 & 818 & 7.82 & $1.38(1.04-1.83)^{*}$ & $1.61(1.21-2.15)^{* *}$ \\
\hline$>2-\leq 3 \mathrm{yrs}$ & 158 & 3044 & 5.19 & 42 & 726 & 5.78 & $1.11(0.79-1.57)$ & $1.22(0.86-1.72)$ \\
\hline$>3$ yrs & 434 & 9948 & 4.36 & 129 & 2643 & 4.88 & $1.13(0.93-1.37)$ & $1.23(1.0-1.5)^{*}$ \\
\hline
\end{tabular}

Rate $=$ mortality rate (per 100 person-years).

Adjusted HR denotes adjusted for sex, age, and comorbidities in Cox proportional hazards regression.

${ }^{*} p<0.05 ;{ }^{* *} p<0.01 ;{ }^{* * *} p<0.001$.

bypass group, with significant difference in the 3-month to 2 -year and $>3$-year postsurgery or stroke periods $(>3$ to $\leq$ 6 months: adjusted HR 1.83, 95\% CI 1.27-2.63, p < 0.01; > 6 to $\leq 12$ months: adjusted HR 1.62, 95\% CI 1.16-2.25, p < $0.01 ;>1$ to $\leq 2$ years: adjusted HR $1.61,95 \%$ CI $1.21-2.15$, $\mathrm{p}<0.01$; and $>3$ years: adjusted HR $1.23,95 \%$ CI 1.0-1.5, $\mathrm{p}<0.05)$.

\section{Discussion \\ Key Results}

Some main findings were noted in this populationbased, nationwide, retrospective cohort study of subsequent stroke (composite of ischemic stroke, hemorrhagic stroke, and death) in patients with symptomatic stenosis and/or occlusion and ischemic stroke who underwent ECIC bypass intervention. First, the bypass group was associated with a lower risk of all types of stroke and subsequent ischemic stroke. Second, this group was associated with higher risk of subsequent hemorrhagic stroke and mortality (Fig. 2). Third, the bypass group was associated with high risk of $\mathrm{CHF}$, whereas the nonbypass group was associated with high risk of AMI.

\section{Interpretation of Results}

EC-IC bypass surgery reroutes blood flow around a blocked or damaged artery to improve or restore blood flow to an ischemic area of the brain. However, any surgery carries risks and EC-IC bypass surgery is no exception. For instance, stroke after EC-IC surgery may occur because of the manipulation and temporary clipping of the arteries in the brain. Recurrent stroke postsurgery could also result from graft failure or failure of adequate blood flow through the newly connected arteries. Furthermore, graft occlusion may also occur after EC-IC surgery, whereby blood clots form inside the donor vessel and block the blood flow. ${ }^{24}$ Such complications, as well as unstable hemodynamics, may be more obvious in the first year following EC-IC surgery. To accurately evaluate the efficacy of EC-IC bypass surgery for the prevention of recurrent stroke, it is necessary to clarify whether recurrent stroke is due to the inefficacy of the surgical intervention itself or to other confounding variables.

Our findings shed some light on these points. The initial stroke risk was higher in the bypass group, but then it tended to decrease after 6 months in the bypass group compared with the nonbypass group. Obviously, the overall risk of subsequent ischemic stroke was mitigated, whereas that of hemorrhage from perioperative risk postsurgery obscured the benefit of this intervention procedure in patients with stroke.

In our study population, the patients in the bypass group had a higher prevalence of CHF history preoperatively, and this might explain the high prevalence of $\mathrm{CHF}$ in this group postsurgery as well. However, it is difficult to explain why the risk of AMI increased in the nonbypass group compared with the bypass group, even though it had a comparable medical history of cardiovascular diseases.

\section{Limitations of the Study}

This retrospective cohort study has several limitations that should be acknowledged. First, there are several causes of ischemic stroke that require EC-IC bypass surgery, such as ICA or common carotid artery stenosis and/ or occlusion, MCA stenosis and/or occlusion, moyamoya disease, basilar artery syndrome, and vertebral artery syndrome. Additionally, the causes of stenosis and/or occlusion of MCA, ICA, and common carotid artery also vary, including atherosclerosis, trauma, dissection, and inflammation. It was hard to identify the different types of stenosis and/or occlusion in this retrospective study; however, we excluded patients with moyamoya disease and syndromes for which EC-IC bypass would not be approved by the National Health Insurance Administration in Taiwan. Although stenosis and/or occlusions due to dissection and inflammation are rare, it is a limitation that we can hardly distinguish between them. To overcome this limitation to a certain extent, we excluded any trauma and malignancy that might influence the outcomes. Second, we could not analyze the cause of death using data from the NHIRD database; thus, it is difficult to determine if the higher mortality was associated with stroke. Third, we did not have 
A

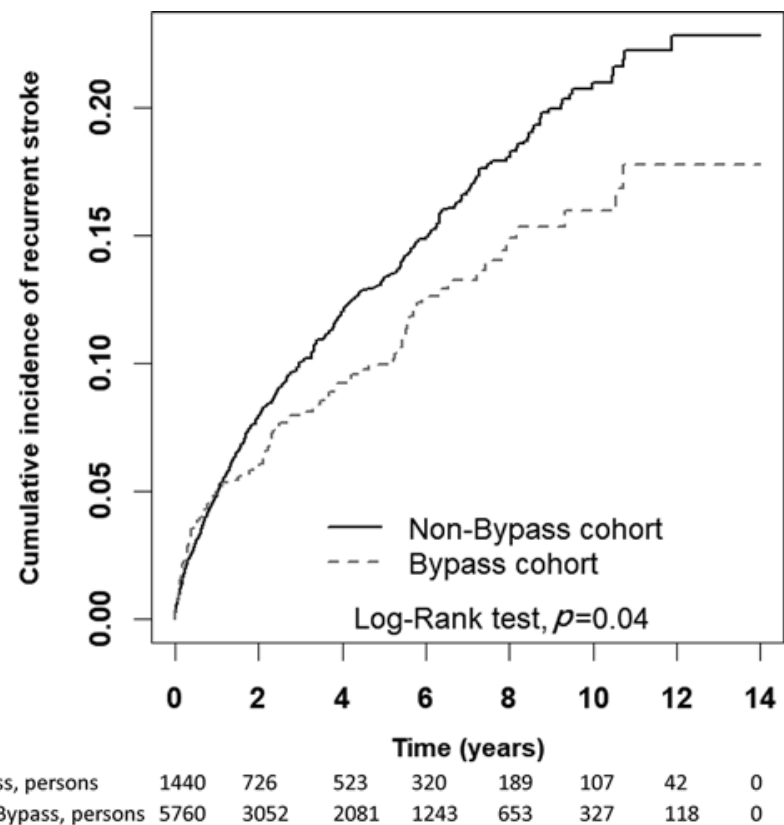

C

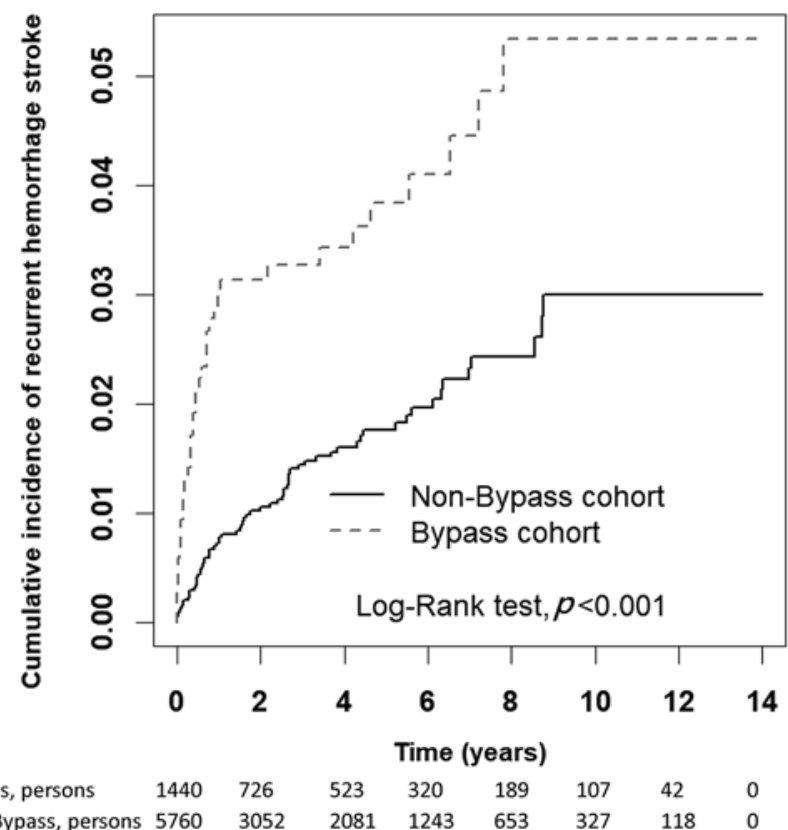

B

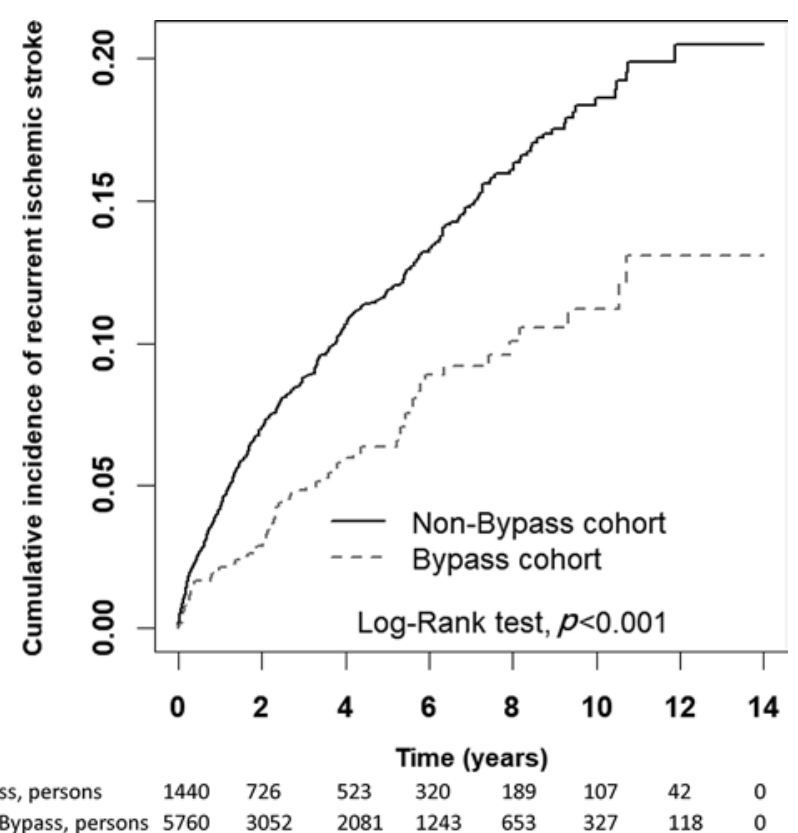

D

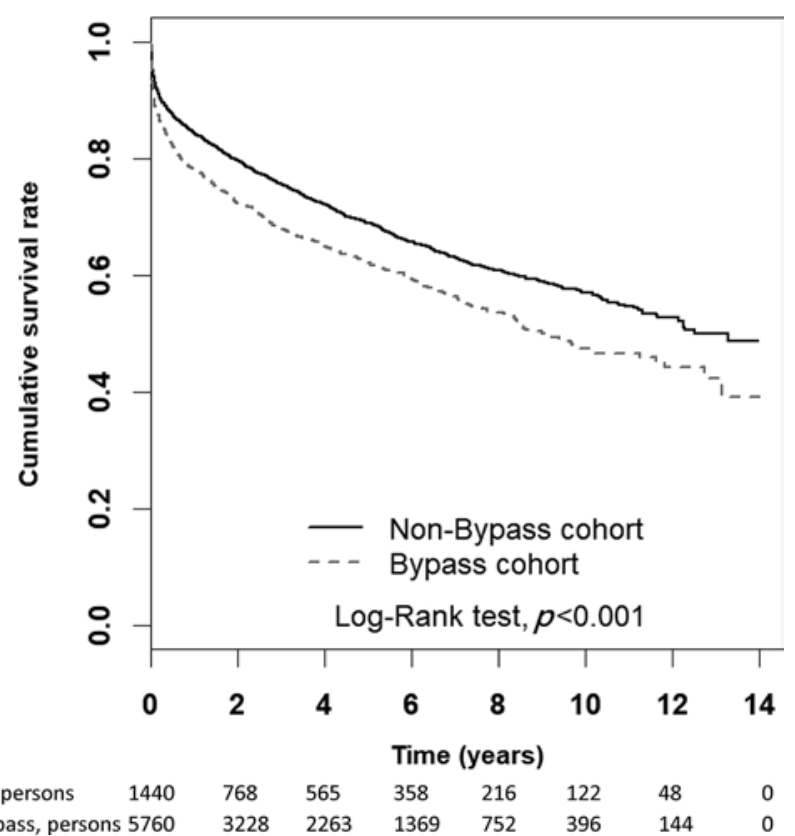

FIG. 2. Graphs showing cumulative incidence of subsequent strokes and mortality. A: Cumulative incidence of subsequent strokes of all types. B: Cumulative incidence of subsequent ischemic stroke. C: Cumulative incidence of subsequent hemorrhagic stroke. D: Cumulative incidence of subsequent mortality. Survival analysis was used to determine the incidence of all types of subsequent strokes, including ischemic stroke and hemorrhagic stroke. Subsequent mortality was analyzed as well.

data of the NIH Stroke Scale and modified Rankin Scale. These factors may have affected the severity of stroke and major vascular outcomes. In our clinical practice experience, the population baselines between cohorts are not even and aggressive interventions would only be chosen when patients have very severe symptoms. Fourth, because of the lack of echocardiographic assessment, it was difficult to distinguish cardioembolic from noncardioembolic stroke. Last, this study did not incorporate randomization, which may have resulted in some selection bias.

\section{Conclusions}

Bypass surgery does play an important role in revascularization of the ischemic brain, and overall, the risk of ischemia was reduced while that of hemorrhage was still high after intervention. The high risk of the surgery itself obscures the true benefit in rescuing the brain from ischemia.

Ischemia can be resolved by revascularization, provided that the eligible patients are carefully selected with 
an understanding of the complex relationship between cerebral blood flow and brain metabolism in determining tissue ischemia and stroke risk. Conversely, failure to differentiate between hemodynamic and thromboembolic etiologies and assess hemodynamic insufficiency during patient selection might render the bypass surgery ineffective. Specifically, patients with known risk factors are unlikely to benefit from surgical intervention. Clinicians should therefore individualize their management strategy until additional data are published or further consensus develops. This study highlights the fact that the high risk of bypass surgery obscures the true benefit of revascularization of the ischemic brain and also emphasizes the importance of developing improved surgical techniques to treat these high-risk patients. Future studies should assess such aspects more rigorously to promote a consensus.

\section{References}

1. Amin-Hanjani S: Cerebral revascularization: extracranialintracranial bypass. J Neurosurg Sci 55:107-116, 2011

2. Biswas A, Samadoni AE, Elbassiouny A, Sobh K, Hegazy A: Extracranial to intracranial by-pass anastomosis: Review of our preliminary experience from a low volume center in Egypt. Asian J Neurosurg 10:303-309, 2015

3. Chater N: Results of neurosurgical microvascular extracranial-intracranial bypass for stroke: a decade of experience. West J Med 138:531-533, 1983

4. Cheng CL, Kao YH, Lin SJ, Lee CH, Lai ML: Validation of the National Health Insurance Research Database with ischemic stroke cases in Taiwan. Pharmacoepidemiol Drug Saf 20:236-242, 2011

5. Derdeyn CP, Grubb RL Jr, Powers WJ: Indications for cerebral revascularization for patients with atherosclerotic carotid occlusion. Skull Base 15:7-14, 2005

6. EC/IC Bypass Study Group: Failure of extracranial-intracranial arterial bypass to reduce the risk of ischemic stroke. Results of an international randomized trial. N Engl J Med 313:1191-1200, 1985

7. Goldring S, Zervas N, Langfitt T: The Extracranial-Intracranial Bypass Study. A report of the committee appointed by the American Association of Neurological Surgeons to examine the study. N Engl J Med 316:817-820, 1987

8. Grubb RL Jr, Derdeyn CP, Fritsch SM, Carpenter DA, Yundt $\mathrm{KD}$, Videen TO, et al: Importance of hemodynamic factors in the prognosis of symptomatic carotid occlusion. JAMA 280:1055-1060, 1998

9. Hänggi D, Steiger HJ, Vajkoczy P: The role of MCA-STA bypass surgery after COSS and JET: the European point of view. Acta Neurochir Suppl 119:77-78, 2014

10. Hankey GJ, Warlow CP: Prognosis of symptomatic carotidartery occlusion. An overview. Cerebrovasc Dis 1:245-256, 1991

11. Hsiao FY, Yang CL, Huang YT, Huang WF: Using Taiwan's National Health Insurance Research databases for pharmacoepidemiology research. J Food Drug Analysis 15:99-108, 2007

12. Kataoka H, Miyamoto S, Ogasawara K, Iihara K, Takahashi JC, Nakagawara J, et al: Results of prospective cohort study on symptomatic cerebrovascular occlusive disease showing mild hemodynamic compromise [Japanese ExtracranialIntracranial Bypass Trial (JET)-2 Study]. Neurol Med Chir (Tokyo) 55:460-468, 2015

13. Klijn CJ, Kappelle LJ: Haemodynamic stroke: clinical features, prognosis, and management. Lancet Neurol 9:10081017,2010

14. Klijn CJ, Kappelle LJ, Tulleken CA, van Gijn J: Symptomatic carotid artery occlusion. A reappraisal of hemodynamic factors. Stroke 28:2084-2093, 1997

15. Klijn CJ, van Buren PA, Kappelle LJ, Tulleken CA, Eikelboom BC, Algra A, et al: Outcome in patients with symptomatic occlusion of the internal carotid artery. Eur J Vasc Endovasc Surg 19:579-586, 2000

16. Murai Y, Mizunari T, Umeoka K, Tateyama K, Kobayashi S, Teramoto A: Radial artery grafts for symptomatic cavernous carotid aneurysms in elderly patients. Neurol India 59:537541, 2011

17. Powers WJ, Clarke WR, Grubb RL Jr, Videen TO, Adams HP Jr, Derdeyn CP: Extracranial-intracranial bypass surgery for stroke prevention in hemodynamic cerebral ischemia: the Carotid Occlusion Surgery Study randomized trial. JAMA 306:1983-1992, 2011 (Erratum in JAMA 306:2672, 2011)

18. Sacquegna T, De Carolis P, Pazzaglia P, Andreoli A, Limoni P, Testa C, et al: The clinical course and prognosis of carotid artery occlusion. J Neurol Neurosurg Psychiatry 45:10371039, 1982

19. Sia SF, Morgan MK: High flow extracranial-to-intracranial brain bypass surgery. J Clin Neurosci 20:1-5, 2013

20. Sundt TM Jr: Was the international randomized trial of extracranial-intracranial arterial bypass representative of the population at risk? N Engl J Med 316:814-816, 1987

21. Sundt TM Jr, Whisnant JP, Fode NC, Piepgras DG, Houser OW: Results, complications, and follow-up of 415 bypass operations for occlusive disease of the carotid system. Mayo Clin Proc 60:230-240, 1985

22. Tsai ST, Yen PS, Wang YJ, Chiu TL: Superficial temporal artery-middle cerebral artery bypass for ischemic atherosclerotic middle cerebral artery disease. J Clin Neurosci 16:1013-1017, 2009

23. Vilela MD, Newell DW: Superficial temporal artery to middle cerebral artery bypass: past, present, and future. Neurosurg Focus 24(2):E2, 2008

24. Zuccarello M: Cerebral Bypass Surgery. Cincinnati: Mayfield Brain \& Spine, 2016 (https://www.mayfieldclinic.com/ PDF/PE-CerebralBypass.pdf) [Accessed January 25, 2018]

\section{Disclosures}

This study was supported by the China Medical University Hospital (DMR-106-068), and was partially supported by Taiwan Ministry of Health and Welfare Clinical Trial Center (MOHW107-TDU-B-212-123004), China Medical University Hospital, Academia Sinica Stroke Biosignature Project (BM10701010021), MOST Clinical Trial Consortium for Stroke (MOST 106-2321-B-039-005), Tseng-Lien Lin Foundation (Taichung, Taiwan), and Katsuzo and Kiyo Aoshima Memorial Funds (Japan), and CMU under the Aim for the Top University Plan of the Ministry of Education, Taiwan. The funders had no role in the study design, data collection and analysis, decision to publish, or preparation of the manuscript.

\section{Author Contributions}

Conception and design: XX Chen. Acquisition of data: XX Chen, Lin. Analysis and interpretation of data: XX Chen, KF Chen. Drafting the article: XX Chen. Critically revising the article: $\mathrm{CC}$ Chen, XX Chen, KF Chen, Wei, Chiu. Reviewed submitted version of manuscript: XX Chen, KF Chen. Approved the final version of the manuscript on behalf of all authors: CC Chen. Statistical analysis: Lin, Su. Administrative/technical/material support: CC Chen, Lai, Wei, Peng, Chiu, Shieh. Study supervision: CC Chen, Shieh.

\section{Correspondence}

Chun-Chung Chen: China Medical University Hospital, Taichung, Taiwan.cck36701@gmail.com. 